\title{
2- $\mu m$ hybrid laser emitter for future carbon dioxide space-borne lidar measurement
}

Julien Lahyani, Nicolas Cézard, Benoit Faure, Fabien Gilbert, Julien Le Gouët

Julien Lahyani, Nicolas Cézard, Benoit Faure, Fabien Gilbert, Julien Le Gouët, "2- $\mu \mathrm{m}$ hybrid laser emitter for future carbon dioxide space-borne lidar measurement," Proc. SPIE 11852, International Conference on Space Optics - ICSO 2020, 118521W (11 June 2021); doi: 10.1117/12.2599328

SPIE Event: International Conference on Space Optics - ICSO 2021, 2021, Online Only 


\section{International Conference on Space Optics-ICSO 2020}

Virtual Conference

30 March-2 April 2021

Edited by Bruno Cugny, Zoran Sodnik, and Nikos Karafolas
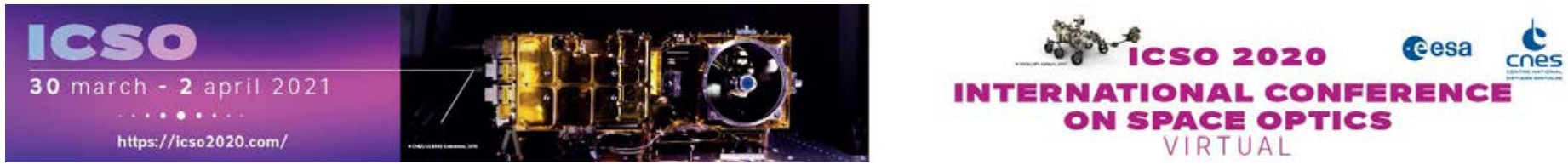

\section{2-um hybrid laser emitter for future carbon dioxide space-borne lidar measurement}

\section{Cesa issopreseatings denes}




\title{
2- $\mu \mathrm{m}$ hybrid laser emitter for future carbon dioxide space-borne lidar measurement
}

\author{
Julien Lahyani ${ }^{\mathrm{a}^{*}}$, Nicolas Cézard ${ }^{\mathrm{b}}$, Benoit Faure ${ }^{\mathrm{c}}$, Fabien Gibert ${ }^{\mathrm{d}}$, and Julien Le Gouët ${ }^{\mathrm{a}}$ \\ aDOTA, ONERA, Université Paris Saclay, F-91123 Palaiseau, France \\ ${ }^{\mathrm{b}}$ ONERA/DOTA, Université de Toulouse, F-31055 Toulouse, France \\ ${ }^{\mathrm{C}} \mathrm{CNES}, 18$ av. Edouard. Belin, 31401 Toulouse, France \\ ${ }^{\mathrm{d}}$ Laboratoire de Météorologie Dynamique (LMD), Centre National de Recherche Scientifique (CNRS), \\ Ecole Polytechnique, FR-91128 Palaiseau Cedex, France
}

\begin{abstract}
We are developing a DIfferential Absorption Lidar (DIAL) for $\mathrm{CO}_{2}$ remote sensing using coherent detection, based on a pulsed hybrid laser emitter at $2.05 \mu \mathrm{m}$. In the $2 \mu \mathrm{m}$ region, the $\mathrm{R} 30 \mathrm{CO}_{2}$ absorption line has been identified as one of the most promising for space-borne DIAL instrument to provide precise sounding of the atmospheric boundary layer where $\mathrm{CO}_{2}$ sources and sinks are located. In this paper, we present the Tm-doped all-fiber part of the emitter and we demonstrate its ability to measure the $\mathrm{CO}_{2}$ Volume Mixing Ratio (VMR) in the atmosphere. The lidar setup is presented, including the telescope, the heterodyne detection system and a spectral reference system used to infer the absolute frequency of the laser and the ON-line/OFF-line energy difference. We investigate an Integrated-Path-DIAL measurement on a range of $426 \mathrm{~m}$. Results compare well with an in-situ reference sensor (Picarro probe), and the estimate statistics show good agreement with theoretical error calculations.

This lidar demonstrator has been called HELENA (Hybrid Emitter Lidar for ENvironmetal Applications). Once completed, it will feature an hybrid emitter combining a semiconductor laser, a high-power Tm-doped fiber amplifier stage, and a final Ho:YLF crystal single-pass amplification stage. This combination aims at benefitting of the versatility, compactness and alignment easiness of a fiber amplifier on one hand, and of the high-peak power attainable with a solid-state amplifier on the other hand. It could allow overcoming the traditional Brillouin limit met in fiber amplifiers, without sacrificing much in compactness and robustness.
\end{abstract}

Keywords: Laser, Lidar, DIAL, Hybrid amplifier

\section{INTRODUCTION}

Carbon dioxide $\left(\mathrm{CO}_{2}\right)$ is acknowledged as the most important anthropogenic greenhouse gas in the atmosphere. Its impact on future climate evolution still suffers some uncertainties ${ }^{1}$. To improve our understanding of the $\mathrm{CO}_{2}$ life cycle, and open ways to control anthropogenic emissions, it is necessary to monitor $\mathrm{CO}_{2}$ sources and sinks fluxes, at a local scale with groundbased instruments, and if possible, at the global scale with space-borne instruments.

Active sensors are especially attractive for such tasks, as they can provide increased space-time coverage and precision ${ }^{2}$ compare to passive sensors. Several lidar systems based on high energy solid-state lasers already demonstrated good capabilities for $\mathrm{CO}_{2}$ monitoring at $2 \mu \mathrm{m}^{3-6}$ and $1.5 \mu \mathrm{m}^{7-10}$, using DIfferential Absorption Lidar (DIAL) or Integrated Path Differential Absorption (IPDA). However, bulk architectures generally involve large numbers of free-space optics that can bring substantial thermal and mechanical problems. Intrinsically, all-fiber laser architectures alleviate those issues. However, 
the well-known Stimulated Brillouin Scattering (SBS) limitation makes difficult reaching the required peak power for a space-borne mission (tens to hundreds of $\mathrm{kW}$ ).

Considering the interest of fiber laser for $\mathrm{CO}_{2}$ space-borne lidar, ONERA, Laboratoire de Météorologie Dynamique (CNRS/LMD), and Centre National d'Etudes Spatiales (CNES) have decided in 2018 to start a two-steps research program. The first step consists in developing a high peak-power, narrow-linewidth, all-fiber pulsed laser source at $2.05 \mu \mathrm{m}$, suitable for standalone $\mathrm{km}$-range ground-based $\mathrm{CO}_{2} /$ wind measurement using coherent detection ${ }^{11}$. The working wavelength has been chosen to match the $\mathrm{R} 30 \mathrm{CO}_{2}$ absorption line which has been identified in previous studies has one of the most promising for space-borne missions ${ }^{12}$. In a second and future step, the fiber source will be combined with a solid-state amplifier for peakpower upscaling. The resulting hybrid fiber/solid-state laser source could represent an attractive trade-off combining high peak-power and limited free-space optics complexity, as required for future space-borne lidar missions.

In the first part, we report the most important results of the all-fiber part of the laser and we derive from the classical DIAL equations, the expected order of magnitude for the random error on the $\mathrm{CO}_{2}$ Volume Mixing Ratio (VMR) with heterodyne and direct detection. In the second part, we describe our lidar design, the method to infer essential parameters for DIAL operation and we show the preliminary IP-DIAL results obtained. In the last part we explain the objectives of the second step of this research project.

\section{ALL-FIBER LASER ARCHITECTURE AND PERFORMANCE}

\subsection{Architecture}

The amplification chain is shown in Figure 1. The source architecture is based on a Polarization Maintaining (PM) Master Oscillator Fiber Power Amplifier (MOFPA) made of four Thulium Doped Fiber Amplifiers (TDFA) pumped by laser diodes at $793 \mathrm{~nm}$. The MOFPA is alternatively seeded by two narrow-linewidth Distributed Feed-Back Laser Diodes (DFB-LD) using an Optical Switch (OS) with a rate up to $20 \mathrm{kHz}$. The pre-amplifier (TDFA1) delivers a continuous signal. The optical power at its output is split in two parts, one delivering the Local Oscillator (LO) power used to generate the beat note signal for heterodyne detection and one seeding the second amplifier. TDFA1 has been design to preserve as much as possible the optical properties of the seeders to ensure optimal detection performance ${ }^{13}$. An Acousto-Optic Modulator (AOM) shapes the signal into pulses and adds an optical frequency offset of $80 \mathrm{MHz}$ for heterodyne detection. At the TDFA2 output, a MachZehnder Electro Optic amplitude Modulator (EOM) is used as a time-gated attenuator to filter amplified AOM parasitic spikes and the inter-pulse Amplified Spontaneous Emission (ASE). TDFA3 output peak power is limited by Stimulated Brillouin Scattering (SBS). Its output is filtered by a High Reflectivity Fiber Bragg Grating (HR-FBG) centered on $2051.2 \mathrm{~nm}$ with a width of $1 \mathrm{~nm}$. It permits to remove the large ASE component that can degrade the amplification efficiency at the signal wavelength in last amplifier. The last amplifier (TDFA4) is made with Large Mode Area (LMA) fiber that is longitudinally stretched to overcome the SBS limit. The laser output is spliced to a pigtail which made easier the collimation. For more details on the fiber laser source, see ${ }^{11,14}$.

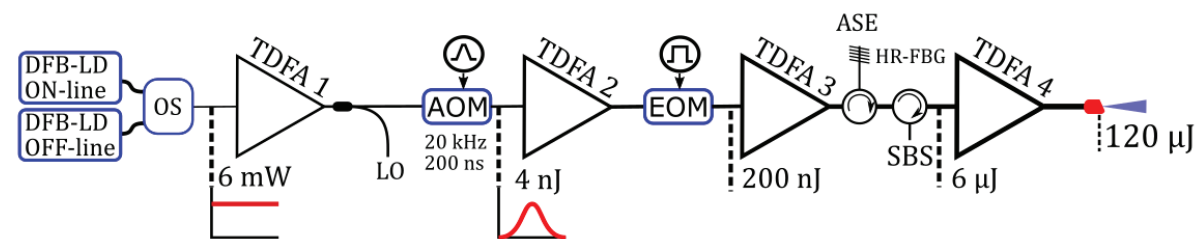

Figure 1: All-fiber MOPA architecture. DFB-LD: Distributed Feedback Laser Diode, OS: Optical Switch, LO: Local Oscillator, TDFA:

Thulium Doped Fiber Amplifier, AOM: Acousto-Optic Modulator, EOM: Electro-Optic Modulator, ASE: Amplified Spontaneous Emission, HR-FBG: High Reflectivity Fiber Bragg Grating, SBS: Stimulated Brillouin Scattering

\subsection{Laser performance}

In this part, we summarized the most important feature of the fiber laser presented in section 2.1 for DIAL/Doppler application. In Table 1, main optical feature are shown 
Table 1 : Main laser optical features

\begin{tabular}{|l|l|}
\hline Feature & Results in this study \\
\hline Laser wavelength & $2.05 \mu \mathrm{m}$ \\
\hline Spectral tunability & $70 \mathrm{GHz}(1 \mathrm{~nm})$ \\
\hline Pulse energy & $120 \mu \mathrm{J}$ \\
\hline Peak - Average power & $600 \mathrm{~W}-2.4 \mathrm{~W}$ \\
\hline Pulse duration & $200 \mathrm{~ns}$ \\
\hline PRF & $20 \mathrm{kHz}$ \\
\hline ON-OFF switch rate & Up to $20 \mathrm{kHz}$ \\
\hline
\end{tabular}

The laser wavelength is center on $2051.2 \mathrm{~nm}$ and tunable over $1 \mathrm{~nm}$ (i.e. $70 \mathrm{GHz}$ ) using the temperature setting of the seeder. The tuning range is limited by the HR-FBG width. It fully covers the R30 absorption line allowing the use of the side band for DIAL operations. In this study, the Pulse repetition Rate (PRF) and the pulse duration are set to $20 \mathrm{kHz}$ and $200 \mathrm{~ns}$ respectively. The laser energy is $120 \mu \mathrm{J}$ which corresponds to $600 \mathrm{~W} / 2.4 \mathrm{~W}$ peak/average power. This working point is slightly under the SBS threshold in order to limit the warming of the pump diodes. The switching rate between the ON-line and OFF-line wavelengths is up to $20 \mathrm{kHz}$ for pulse-to-pulse switching. Fast wavelength switching is required to keep high atmospheric correlation between ON and OFF signals. For the atmosphere to be considered as 'frozen', a switching rate of $1 \mathrm{kHz}$ or more is typically required ${ }^{15}$ (for space-borne operation, the requirement is higher since the satellite is moving very fast. In current solid-state lidar designs, the inter-pulse time between ON and OFF laser shots is typically $250 \mu \mathrm{s}$, which sets at $4 \mathrm{kHz}$ the $\mathrm{ON}-\mathrm{OFF}$ switching rate requirement ${ }^{16}$ ). In Table 2 , the main features for lidar operations are summarized.

Table 2 : Laser features for DIAL operation

\begin{tabular}{|l|l|}
\hline Features & Results in this study \\
\hline RIN (around AOM frequency shift) & $-160 \mathrm{~dB} / \mathrm{Hz}$ \\
\hline Spectral Linewidth & $<5 \mathrm{MHz}$ \\
\hline $\begin{array}{l}\text { Signal -LO beat frequency } \\
\text { stability @ 10 ms - 10 s }\end{array}$ & $100 \mathrm{kHz}$ \\
\hline Beam quality $\mathbf{M}^{\mathbf{2}}$ & 1,12 \\
\hline $\begin{array}{l}\text { Polarization } \\
\text { Extinction Ratio }\end{array}$ & Linear, PER $>16 \mathrm{~dB}$ \\
\hline Free running frequency drift & $<50 \mathrm{MHz}$ \\
\hline Cross-Talk & $-23 \mathrm{~dB}$ \\
\hline SMSR & $>45 \mathrm{~dB}$ \\
\hline
\end{tabular}

Heterodyne detection required a LO with low RIN. In our scheme, the noise is limited by the shot noise (quantum limit) at $\mathrm{RIN}=-170 \mathrm{~dB} / \mathrm{Hz}$. In this study, we reach $-160 \mathrm{~dB} / \mathrm{Hz}$ which leads to less than $1 \mathrm{~dB}$ loss on the Carrier-to-Noise Ratio (CNR). The spectral linewidth represents another critical issue to reduce the integrated noise. Indeed, narrower is the signal spectrum, lower is the integrated noise and higher is the CNR. In our case, the spectral linewidth is lower than $5 \mathrm{MHz}$. It represents a loss on the CNR of $1 \mathrm{~dB}$ compared to a pure Fourier-transform limited pulse (considering also the DFB-LD 
linewidth of $2 \mathrm{MHz}$ ). The Signal-LO (S-LO) beat frequency stability can involve a broadening of the signal spectrum when averaging shots, and for the reason explained before, it could degrades the CNR. The RMS S-LO stability is $100 \mathrm{kHz}$ at $10 \mathrm{~ms}$ averaging time and follows a root-time decrease up to $10 \mathrm{~s}$. It implies negligible broadening of the averaged signal spectrum. The beam quality $\left(\mathrm{M}^{2}\right)$ denotes the beam divergence and is crucial to maximize the CNR. In our case it represents a loss of $0.5 \mathrm{~dB}$ on the CNR. Last three features in Table 2 play a role on the $\mathrm{CO}_{2}$ measurement bias. The seeder is working in free-running mode and we measured a frequency shift lower than $50 \mathrm{MHz}$ on 10 minutes. It leads to less than $0.2 \%$ bias on the $\mathrm{CO}_{2}$ VMR (at R30 center), but anyway it will be necessary in the futur to lock the DFB-LD frequency ${ }^{17,18}$ to ensure optimal performance. The transmission of the switched off wavelength is called the cross-talk (or spectral purity) and is crucial for DIAL measurement. In heterodyne detection, this feature is relaxed compared to the direct detection. In our case, the cross talk is $-23 \mathrm{~dB}$ on both the $\mathrm{LO}$ and the emitted signal which leads to a bias lower the $0.1 \%$ up to $3 \mathrm{~km}$ of propagation in the atmosphere. The SMSR is also an important issue in the same way as the cross-talk. Our SMSR is higher than $45 \mathrm{~dB}$ and involve negligible VMR bias.

\subsection{Expected lidar performance}

In the perspective of a global monitoring of the $\mathrm{CO}_{2}$ from space, the use of direct detection appears as much more performant to reach space mission goal of $0.1 \%$ VMR relative error. This study being in its first phase and for a reason of simplicity of implementation, we performed a heterodyne detection. It also presents the advantage to allow simultaneous wind monitoring for autonomous $\mathrm{CO}_{2}$ flux rate measurement. However, in this part, we investigate the VMR error for both, heterodyne and direct detection.

The DIAL equation writes ${ }^{19}$ :

$$
V M R=\frac{1}{2 . \Delta z \cdot W F} \ln \left(\frac{P_{O N}(z)}{P_{O F F}(z)} \cdot \frac{P_{O F F}(z+\Delta z)}{P_{O N}(z+\Delta z)}\right)
$$

Where WF is the Weighting Function (in $\mathrm{ppm}^{-1} \cdot \mathrm{m}^{-1}$ ), $\Delta \mathrm{z}$ is the range resolution (distance to hard target for IP-DIAL measurement) and $\mathrm{P}_{\mathrm{ON} / \mathrm{OFF}}$ is the returned signal power. The log expression represents the slope of the optical depth i.e. the absorption coefficient. The VMR error can be expressed as:

$$
\sigma_{V M R}=\frac{1}{2 . \Delta z \cdot W F \cdot \sqrt{N_{a v g}}} \cdot \sqrt{\frac{\sigma^{2}\left(P_{O F F}\right)}{P_{O F F}^{2}}+\frac{\sigma^{2}\left(P_{O N}\right)}{P_{O N}^{2}}-2 \cdot \rho_{P_{O N}, P_{O F F}} \cdot \frac{\sigma\left(P_{O N}\right) \cdot \sigma\left(P_{O F F}\right)}{P_{O N} \cdot P_{O F F}}}
$$

Where $N_{\text {avg }}$ is the number of VMR values averaged, $\sigma$ is the standard deviation and $\rho$ is the correlation coefficient, $\rho_{X, Y}=\operatorname{cov}(X, Y) /\left(\sigma_{X} . \sigma_{Y}\right)$ with $\operatorname{cov}$ the covariance function. In this equation, we can identify the Signal-to-noise Ratio as $\mathrm{SNR}_{\mathrm{P}}=\mathrm{P} / \sigma(\mathrm{P})$. In heterodyne detection, the $\mathrm{SNR}$ can be written ${ }^{3}$ : 


$$
\operatorname{SNR}_{P}=\frac{\sqrt{N_{\text {pulses }} \cdot M_{t}}}{\left(1+\frac{1}{\langle C N R\rangle}\right)}
$$

Where $N_{\text {pulses }}$ is the number of shots averaged to calculate a single returned power $P, M_{t}$ is the number of independent speckle patterns and CNR is the Carrier-to-Noise Ratio. In practice, $M_{t}$ is close to unity when the range gate equals to the laser coherence length. For a single shot $\left(\mathrm{N}_{\text {pulses }}=1\right)$, the $\mathrm{SNR}_{\text {pulse }}$ is close to the unity in heterodyne detection. For this reason, it is better to use heterodyne detection with high PRF $(>1 \mathrm{kHz})$ as it allows a fast accumulation to increase the SNR and then reduce the VMR error. Regarding the energy of the fiber laser of $120 \mu \mathrm{J}$, the CNR will be usually lower than $0 \mathrm{~dB}$ for atmospheric signal and higher than $10 \mathrm{~dB}$ up to $5 \mathrm{~km}$ on a hard target.

In direct detection, the SNR for a single shot can be higher than 1 as the speckle noise can be largely averaged by the detector area $^{20}$. For space-borne measurement, as the number of averaged shot is limited, the use of direct detection appears preferable. In Killinger et al. ${ }^{21}$, the inter-comparison between heterodyne and direct detection shows, in same conditions, a SNR five times higher in direct detection.

On Figure 2 is plotted the VMR standard deviation assuming $\mathrm{SNR}_{\mathrm{ON}}=\mathrm{SNR}_{\mathrm{OFF}}$ (case where the distance of propagation is not sufficient to involve important absorption or case where ON-line and OFF-line signals energy are chosen to compensate the absorption), $\mathrm{WF}=1.6 .10^{-6} \mathrm{ppm}^{-1} \cdot \mathrm{m}^{-1}$ (On-line signal at the R30 centre and no pressure/temperature variation on the line of sight) and the correlation degree equals to 0 function of the SNR for a single pulse ( $\mathrm{SNR}_{\text {pulse, }}$, horizontal axis) and the product $\mathrm{N}_{\mathrm{avg}}{ }^{1 / 2} . \Delta \mathrm{z}$ (vertical axis) which is representative to the space-time resolution.

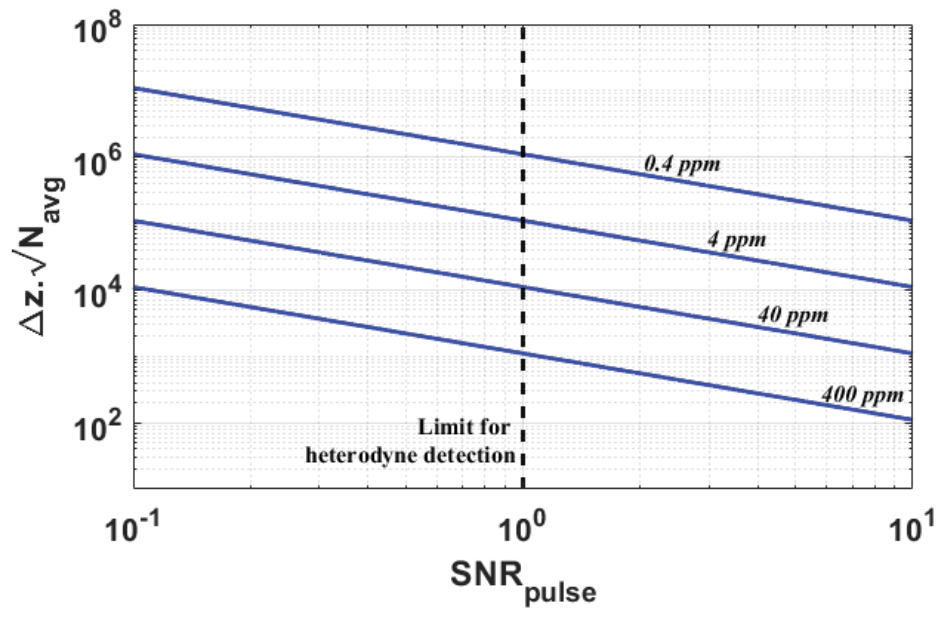

Figure 2 : VMR error as function of the single pulse SNR and the product $\mathrm{N}_{\text {avg }}{ }^{1 / 2} \cdot \Delta \mathrm{z}$

The $\mathrm{CO}_{2}$ concentration in the atmosphere is around $400 \mathrm{ppm}$. Plotted blue lines stand for the relative errors of $100 \%, 10 \%$, $1 \%$ and $0.1 \%$ (precision goal for spatial mission). For $1 \mathrm{~km}$ integrated path and 100 pairs of shot averaged, an heterodyne measurement can reach $10 \%$ relative error while a direct detection can reach $2 \%$ in the same condition regarding the performance reported $\mathrm{in}^{21}$. Moreover, if there is no relative displacement between hard target and the emitter, the speckle pattern is unchanged and the statistical error decrease slower in time. In the assessment for the A-Scope mission ${ }^{2}$, some feasibility studies are detailed. 


\section{3. $\mathrm{CO}_{2}$ SENSING DEMONSTRATION USING HETERODYNE DETECTION}

All results presented in following sub-sections have been made at $120 \mu \mathrm{J}, 200 \mathrm{~ns}, 20 \mathrm{kHz}$ and pulse-to-pulse ON/OFF switching rate. In the Emission/Reception module (E/R), the emitted light is collimated and goes through a Brewster angle plate that transmits the p-polarization and reflects the s-polarization. The residual signal on Figure 3 corresponds to the spolarized part of the field. It is used for spectral reference and calculation of the energy ratio (see section 3.2). The PER is higher than $16 \mathrm{~dB}$, thus most of the flux is transmitted to the telescope. The emitted beam has a diameter of $5.3 \mathrm{~cm}$ at $1 / \mathrm{e}^{2}$. The quarter wave plate in the telescope change the polarization by $90^{\circ}$ after a round trip to reflect on the Brewster plate the backscattered signal.

Signal and LO are combined by a 95:5 coupler to the detector. The $95 \%$ arm is for the signal. The detector is a PIN photodiode with $150 \mathrm{MHz}$ bandwidth, delivering $1.2 \mathrm{~A} / \mathrm{W}$ and with a NEP of $8 \mathrm{pW} . \mathrm{Hz}^{-1 / 2}$ around the AOM frequency shift . The electronic signal is then band-pass filtered between 40 and $100 \mathrm{MHz}$. The analog signal is digitized by a 12 bits ADC at $250 \mathrm{MHz}$. The ADC is triggered by a TTL signal synchronized with the AOM command.

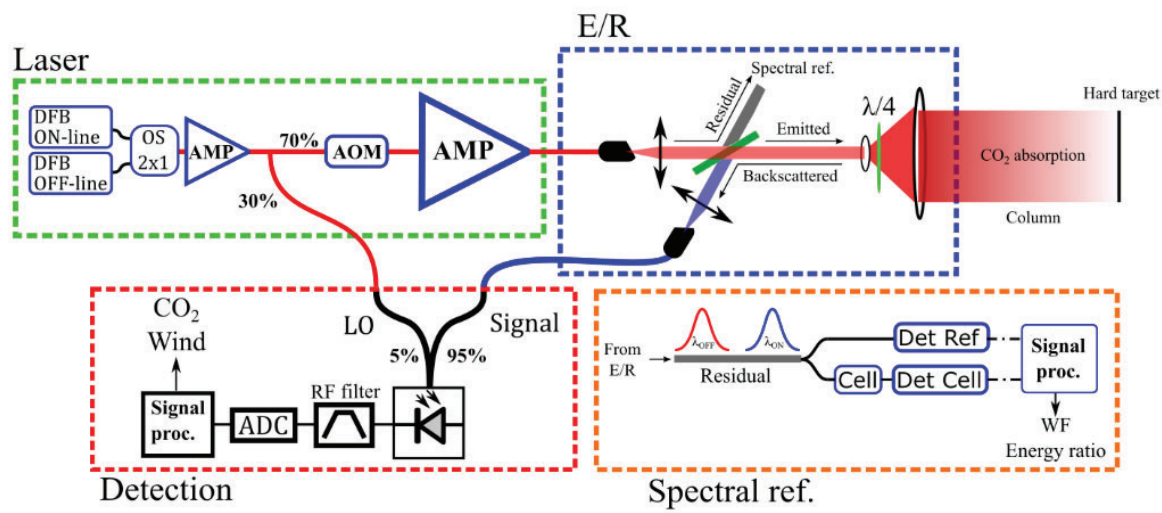

Figure 3 : Lidar design

\subsection{Signal processing}

The ON-line and OFF-line signals are handled separately for the power estimations. A sliding square gate of 200 ns (i.e. the coherence time of the pulse) is applied to the time-domain signal. The Power Spectral Density (PSD) is calculated for each distance-gate using a Fast Fourrier Transform (FFT). To smooth the spectrum we average $\mathrm{N}_{\text {pulses }}$ PSDs. The last sample of each shot is used as the noise PSD (called PSD ${ }_{\text {noise}}$ ) and is subtracted to previous samples. Then the noise-subtracted PSD, called PSD het, is numerically filtered around the peak signal with a $20 \mathrm{MHz}$ square gate and integrated to estimate the return signal power $\mathrm{P}$. The CNR is calculated by dividing the power P by the noise using the same numerical filter. The procedure is the same as the one detailed $\mathrm{in}^{22}$.

\subsection{Absolute spectral reference system and ON-line/OFF-line energy difference monitoring for DIAL operation}

The spectral reference system is shown on Figure 3 (Spectral ref.). The residual beam from the Brewster plate is used to determine the energy difference between ON-line and OFF-line pulses and to estimate the absolute frequency of the laser. The light is fiber-coupled and separated in two parts. The first part goes directly to a DC-coupled detector and allows the determination of ON/OFF energy difference (value $\mathrm{K}$ in Eq.(4)). The second path goes through a gas cell filled with pure $\mathrm{CO}_{2}$. Then, it is possible to calculate the differential absorption of the cell as the $\mathrm{ON}$ and OFF-line pulses are consecutive.

Once digitized, each signal is averaged to improve the SNR. To calculate the transmission, we set the OFF-line signal at $18.8 \mathrm{GHz}$ apart from R30 absorption line center. The tuning precision of our current and temperature drivers gives a resolution of $+/-250 \mathrm{MHz}$ considering the measured DFB-LD wavelength sensitivity. It involves a potential bias on the ON- 
line frequency of $50 \mathrm{MHz}$. The relative error on the WF is then lower than $0.2 \%$. We used the HITRAN database and a local temperature sensor to compute the cell transmission. In a future phase, a frequency locking unit will be added to the system.

In the preliminary test presented in the following sub-section, WF and K are calculated by averaging 1000 pairs of shots. The $\mathrm{K}$ value is given with a relative random error lower than $1 \%$ over the averaging time of $10 \mathrm{~ms}$ to $10 \mathrm{~s}$. The ON-line frequency is given with a random error of $25 \mathrm{MHz}$ which represents a relative random error on the WF lower than $0.1 \%$.

\subsection{IP-DIAL}

To perform an IP-DIAL measurement, we pointed the lidar on a static target located at $426 \mathrm{~m}$. The IP-DIAL equation writes:

$$
V M R_{I P}=\frac{1}{2 \cdot L \cdot W F} \ln \left(\frac{P_{\text {OFF }}(L)}{P_{O N}(L)} \cdot K\right)
$$

This equation is the same as Eq.(1) using $\mathrm{z}=0$ and $\Delta \mathrm{z}=\mathrm{L}$ where $\mathrm{L}$ is the distance to the hard target and $\mathrm{K}$ the energy difference between the $\mathrm{ON}$ and $\mathrm{OFF}$ signals $\left(\mathrm{K}=\mathrm{P}_{\mathrm{ON}}(0) / \mathrm{P}_{\mathrm{OFF}}(0)\right)$ determined using the spectral reference system. The WF for this measurements is $1,57.10^{-6} \mathrm{ppm}^{-1} \cdot \mathrm{m}^{-1}$ slightly detuned from the R30 absorption line center. As a reference, we had a Picarro probe close to the lidar giving the VMR with a sub-ppm precision.

The Figure 4 (a) represents the VMR Allan deviation performed with a VMR time series where $\mathrm{N}_{\text {pulses }}=100$ shots and $\mathrm{N}_{\text {avg }}=$ 1. The minimum time-resolution is then $10 \mathrm{~ms}$. On Figure 4 (b), the time series VMR (doted, red) is plotted with a time averaging of 2 s i.e. $2 \cdot 10^{4}$ pairs of shots $\left(\mathrm{N}_{\text {pulses }}=100\right.$ and $\left.\mathrm{N}_{\mathrm{avg}}=200\right)$. The blue line is the Picarro probe data.

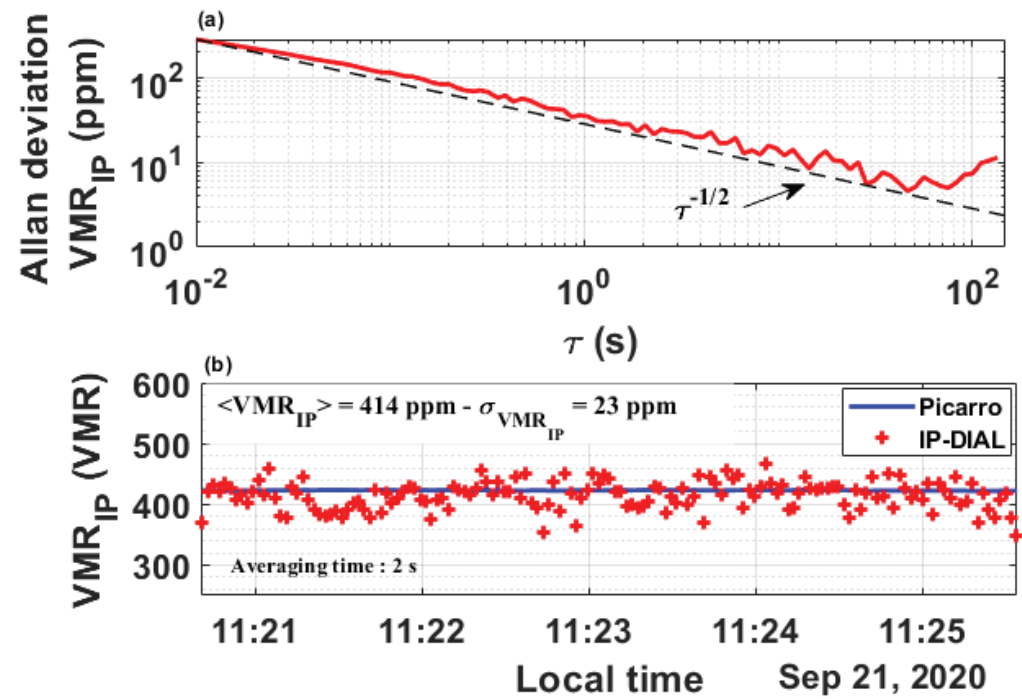

Figure 4 : (a) Allan deviation on the VMR (red) and -1/2 slope representing the stationary behavior (dashed, black), (b) VMR measurement of the Picarro probe (blue) and measured VMR by the lidar (doted, red)

The Allan deviation follows a $\tau^{-1 / 2}$ slope i.e. a stationary behavior over the measurement time of 5 minutes. The average value of the VMR is $414 \mathrm{ppm}$ and the value given by the Picarro probe is $422.5 \mathrm{ppm}$. As the measurements are not co-located, we 
are not able (currently) to determine the absolute precision of our instrument. However, it suggests that there is no major instrumental default. The statistical features calculated and observed for this measurement are summarized in Table 3.

Table 3 : IP-DIAL error analysis

\begin{tabular}{|c|c|c|c|c|c|c|c|c|}
\hline \multirow{2}{*}{\multicolumn{2}{|c|}{$\begin{array}{c}\text { Observed On-line } \\
\text { signal }\end{array}$}} & \multirow{2}{*}{\multicolumn{2}{|c|}{$\begin{array}{c}\text { Observed Off-line } \\
\text { signal }\end{array}$}} & \multirow{3}{*}{$\begin{array}{c}\rho_{P_{\text {ON }}, P_{\text {OFF }}} \\
\mathbf{N}_{\text {pulses }}=\mathbf{1 0 0} \\
\text { Observed }\end{array}$} & \multicolumn{4}{|c|}{ IP-DIAL error } \\
\hline & & & & & \multicolumn{2}{|c|}{$N_{\text {pulses }}=100, N_{\text {avg }}=1$} & \multicolumn{2}{|c|}{$N_{\text {pulses }}=100, N_{\text {avg }}=200$} \\
\hline CNR & $\mathrm{SNR}_{\mathrm{P}}$ & CNR & $\mathrm{SNR}_{\mathrm{P}}$ & & Calculated & Observed & Calculated & Observed \\
\hline $26,64 \mathrm{~dB}$ & 2.0 & $29,02 \mathrm{~dB}$ & 2.0 & 0.64 & $316 \mathrm{ppm}$ & $309 \mathrm{ppm}$ & $22 \mathrm{ppm}$ & $23 \mathrm{ppm}$ \\
\hline
\end{tabular}

The SNR of $\mathrm{P}_{\mathrm{ON}}$ and $\mathrm{P}_{\mathrm{OFF}}$ are similar as the distance of propagation does not significantly absorb the ON-line signal. Furthermore, the hard target signal is strong enough to limit the SNR by the speckle noise. The observed correlation degree of 0.64 is a consequence of high PRF and high switching rate which keep 'frozen' the atmosphere and the target reflectivity between ON-line and OFF-line signals. The observed IP-DIAL error is in good agreement with the calculations.

\section{HYBRID EMITER}

Looking forward for power-scaling of the fiber laser source, the second step of this research project aims at adding a single pass amplification stage in a Ho:YLF crystal. Such an amplifier could overcome the peak power limit encountered in optical fibers due to the Stimulated Brillouin Scattering (SBS). Ho:YLF crystals are usually pumped around $1940 \mathrm{~nm}$ using Tmdoped pump fiber lasers. This kind of high power, continuous wave fiber laser is available commercially and shows electrooptic efficiency higher than $10 \%$. Moreover, the considered single-pass scheme is expected to be much more robust than a cavity, which would strongly alleviates mechanical problems encountered with such cavities.

The experimental work is in progress. The resonant pumping is performed by a $50 \mathrm{~W}$ continuous wave laser emitting at $1940 \mathrm{~nm}$ with a linewidth of $0.4 \mathrm{~nm}$. Both the signal and the pump beams are focused in the crystal with an adjustable waist of $600 \mu \mathrm{m}$ to $1000 \mu \mathrm{m}$. According to the simulations, lower is the beam waist, the more efficient is the amplification. However, the beam divergence and the laser-induced damage threshold of the crystal are the main limits. Once combined, the beams go through a $8 \mathrm{~mm}$-long $0.5 \%$ doped Ho:YLF crystal. At $20 \mathrm{kHz}$, the expected gain is one order of magnitude. At lower PRF, the gain is expected to increase, as the upper-state lifetime in a Ho:YLF crystal is about $15 \mathrm{~ms}$. This demonstration could open new perspectives to provide reliable, performant and robust laser architecture for ground-based or space-borne $\mathrm{CO}_{2}$ lidar measurements. The detailed and performance of this experiment will be published in a future paper.

\section{CONCLUSION}

An all-fiber laser source at $2.05 \mu \mathrm{m}$ has been built and used for $\mathrm{CO}_{2}$ DIAL application. The fiber laser provides pulses of $200 \mathrm{~ns}$ with high PRF of $20 \mathrm{kHz}$ and pulse-to-pulse switching rate using electro-optic system. The main laser characteristics involved in lidar performance (random and systematic error) are summarized. A sub-system used for the monitoring of the wavelength and the pulse energy difference is described in order to perform DIAL measurement. This system uses a gas cell filled with pure $\mathrm{CO}_{2}$ at known pressure. In this study, only free-running seeder operation is performed. We demonstrate a first IP-DIAL measurement over 5 minutes of time. A Picarro probe gives an in-situ measurement of the VMR at the lidar location (i.e. zero distance for the DIAL measurement). Even if the inter-comparison is difficult due to possible urban $\mathrm{CO}_{2}$ emission sources on the line-of-sight, only $2 \%$ bias is found with the IP-DIAL measurement. The random error is consistent with the theoretical calculation.

We also presented the next step of this research project, that aims at demonstrating a hybrid laser amplifier, benefiting of the robustness of a fiber architecture combined with the high peak power of a solid-state amplifier. The single-pass architecture of the free-space amplifier should alleviate mechanical issues encountered in bulk architectures. 


\section{REFERENCES}

[1] Pachauri, R. K., Mayer, L. and Intergovernmental Panel on Climate Change, eds., [Climate change 2014: synthesis report], Intergovernmental Panel on Climate Change, Geneva, Switzerland (2015).

[2] Clissold, P. and European Space Agency, eds., [Six candidate Earth Explorer core missions: reports for assessment: ASCOPE, BIOMASS, CoReH²O, FLEX, PREMIER, TRAQ], ESA Communications, Noordwijk, The Netherlands (2008).

[3] Gibert, F., Flamant, P. H., Bruneau, D. and Loth, C., "Two-micrometer heterodyne differential absorption lidar measurements of the atmospheric CO2 mixing ratio in the boundary layer," Appl. Opt., AO 45(18), 4448-4458 (2006).

[4] Ishii, S., Mizutani, K., Fukuoka, H., Ishikawa, T., Philippe, B., Iwai, H., Aoki, T., Itabe, T., Sato, A. and Asai, K., "Coherent $2 \mu \mathrm{m}$ differential absorption and wind lidar with conductively cooled laser and two-axis scanning device," Appl. Opt., AO 49(10), 1809-1817 (2010).

[5] Singh, U. N., Walsh, B. M., Yu, J., Petros, M., Kavaya, M. J., Refaat, T. F. and Barnes, N. P., "Twenty years of Tm:Ho:YLF and LuLiF laser development for global wind and carbon dioxide active remote sensing," Opt. Mater. Express, OME 5(4), 827-837 (2015).

[6] Cadiou, E., Mammez, D., Dherbecourt, J.-B., Gorju, G., Pelon, J., Melkonian, J.-M., Godard, A. and Raybaut, M., "Atmospheric boundary layer $\mathrm{CO} 2$ remote sensing with a direct detection LIDAR instrument based on a widely tunable optical parametric source," Opt. Lett., OL 42(20), 4044-4047 (2017).

[7] Amediek, A., Fix, A., Wirth, M. and Ehret, G., "Development of an OPO system at $1.57 \mu \mathrm{m}$ for integrated path DIAL measurement of atmospheric carbon dioxide," Appl. Phys. B 92(2), 295-302 (2008).

[8] Sakaizawa, D., Kawakami, S., Nakajima, M., Sawa, Y. and Matsueda, H., "Ground-based demonstration of a CO2 remote sensor using a $1.57 \mu \mathrm{m}$ differential laser absorption spectrometer with direct detection," JARS 4(1), 043548 (2010).

[9] Yu, A. W., Abshire, J. B., Storm, M. and Betin, A., "Laser amplifier development for IPDA Lidar measurements of CO 2 from space,” Proc. SPIE 9342, San Francisco, California, United States (2015).

[10] Amediek, A., Ehret, G., Fix, A., Wirth, M., Büdenbender, C., Quatrevalet, M., Kiemle, C. and Gerbig, C., "CHARM$\mathrm{F}$ - a new airborne integrated-path differential-absorption lidar for carbon dioxide and methane observations: measurement performance and quantification of strong point source emission,” Appl. Opt., AO 56(18), 5182-5197 (2017).

[11] Lahyani, J., Le Gouët, J., Gibert, F. and Cézard, N., "2.05- $\mu \mathrm{m}$ all-fiber laser source designed for CO2 and wind coherent lidar measurement," (in press), Appl. Opt., AO 60(15), (2014).

[12] Caron, J., Durand, Y., Bezy, J.-L. and Meynart, R., "Performance modeling for A-SCOPE: a space-borne lidar measuring atmospheric CO 2," presented at SPIE Europe Remote Sensing, 17 September 2009, Berlin, Germany, $74790 \mathrm{E}$.

[13] Cariou, J.-P., Augere, B. and Valla, M., "Laser source requirements for coherent lidars based on fiber technology," Comptes Rendus Physique 7(2), 213-223 (2006).

[14] Lucas, E., Lombard, L., Jaouën, Y., Bordais, S. and Canat, G., "1 kW peak power, 110 ns single-frequency thulium doped fiber amplifier at $2050 \mathrm{~nm}$," Appl. Opt., AO 53(20), 4413-4419 (2014).

[15] Menyuk, N. and Killinger, D. K., "Temporal correlation measurements of pulsed dual CO2 lidar returns," Opt. Lett., OL 6(6), 301-303 (1981).

[16] Gibert, F., Pellegrino, J., Edouart, D., Cénac, C., Lombard, L., Le Gouët, J., Nuns, T., Cosentino, A., Spano, P. and Di Nepi, G., "2- $\mu \mathrm{m}$ double-pulse single-frequency Tm:fiber laser pumped Ho:YLF laser for a space-borne $\mathrm{CO}_{2}$ lidar," Appl. Opt. 57(36), 10370 (2018).

[17] Numata, K., Chen, J. R., Wu, S. T., Abshire, J. B. and Krainak, M. A., "Frequency stabilization of distributedfeedback laser diodes at $1572 \mathrm{~nm}$ for lidar measurements of atmospheric carbon dioxide,” Appl. Opt., AO 50(7), 1047-1056 (2011).

[18] Curtis, E. A., Bradley, T., Barwood, G. P., Edwards, C. S., Wheeler, N. V., Phelan, R., Richardson, D. J., Petrovich, M. N. and Gill, P., "Laser frequency stabilization and spectroscopy at $2051 \mathrm{~nm}$ using a compact CO2-filled Kagome hollow core fiber gas-cell system," Opt. Express, OE 26(22), 28621-28633 (2018). 
[19] Measures, R. M., [Laser remote sensing: fundamentals and applications], Krieger Pub. Co, Malabar, Fla (1992).

[20] Bruneau, D., Gibert, F., Flamant, P. H. and Pelon, J., "Complementary study of differential absorption lidar optimization in direct and heterodyne detections," Appl. Opt. 45(20), 4898 (2006).

[21] Killinger, D. K., Menyuk, N. and DeFeo, W. E., "Experimental comparison of heterodyne and direct detection for pulsed differential absorption CO_2 lidar," Appl. Opt. 22(5), 682 (1983).

[22] Le Méhauté, S., Benoit, P., Cézard, N., Goular, D., Planchat, C., Dolfi-Bouteyre, A., Delbarre, H., Watremez, X. and Valla, M., "All-fibered coherent-differential absorption lidar at $1.645 \mu \mathrm{m}$ for simultaneous methane and wind speed measurements," Lidar Technologies, Techniques, and Measurements for Atmospheric Remote Sensing XIV, U. N. Singh and G. D. Tzeremes, Eds., 2, SPIE, Berlin, Germany (2018). 